\title{
AN EFFICIENT HYBRID SEALED BID AUCTION PROTOCOL
}

\author{
Riham Abdel-Moneim, Sherif El-Kassas, and Hoda Hosny \\ The Department of Computer Science, The American University in Cairo \\ \{riham, sherif,hhosny\}@aucegypt.edu
}

\begin{abstract}
This paper proposes a new sealed bid auction protocol for internet-based auction, which is based on an earlier developed sealed bid auction protocol called the "hash chain sealed bid auction protocol" by Suzuki, Kobayashi, and Morita in [10]. The protocol proposed in this paper depends on a hybrid approach of public key cryptography and one-way hash functions. A hash chain is used to commit the exact bidding price while the range in which this price exists is secured through public key cryptography. This hybrid approach maintains all the prominent features that distinguish the original hash chain protocol such as keeping the secrecy of the losing bids even from the auctioneer and reducing the size of the bid commitment while making the modified hash chain protocol more efficient and practical than the original hash chain protocol that is purely based on one-way hash functions. Furthermore, our approach does not totally depend on public key cryptography, which is very costly. Our approach experiences less network delay at bid opening time and does not require user interaction during bid opening, as in the original hash chain protocol, which increases the practicality and the applicability of the protocol.
\end{abstract}

\section{INTRODUCTION}

In the past five years, the number of virtual markets and trades conducted over the Internet has seen an explosive growth. Online auctions appear to revolutionize the way many goods and services are traded. Auctions have been an established method of commerce for generations since they are the most important market mechanism for setting prices. In an auction, a good can be sold at a price determined through the interaction between buyers and sellers and this forms the market. The Internet provides

The original version of this chapter was revised: The copyright line was incorrect. This has been corrected. The Erratum to this chapter is available at DOI: 10.1007/978-0-387-35586-3_46 
an infrastructure for running auctions much cheaper with many more market participants that are not bounded anymore by their physical location [11].

There has been a lot of research on auctions, especially on sealed bid auctions since they have special security requirements, as illustrated in section 2.2. Sealed bid auction is the type of auction in which the buyers are required to submit their bids by a specified deadline. The auctioneer keeps the bid information secret until the deadline, at which time the bids are evaluated and the winners are declared. However, existing sealed bid auction protocols depend purely either on public key cryptography or on one-way hash functions. Depending purely on any of the two approaches alone has problems. The protocols discussed in [2], [6], and [9] depend purely on public key cryptography to secure bidding information. However, pubic key cryptosystems are computationally expensive and require a lot of communication. The inefficiency of the public key cryptosystems forces the auction methods to limit the number of bidders and the range of bidding prices.

The first protocol that purely depends on one-way hash functions is the one I base my work on, which is the original hash chain sealed bid auction discussed in [10]. This protocol suffers from some problems that reduce its practicality for large-scale sealed bid auctions. The hash chain sealed bid auction protocol requires the bidders to interact with the auctioneer at bid opening time to keep the secrecy of losing bids, which is inconvenient to the bidders. Besides, this interaction with the bidders during opening time increases the time the auctioneer remains idle waiting for the responses from the bidders and increases the network delay experienced during the opening phase. This makes the time complexity of the opening phase high and reduces the practicality of the protocol. Furthermore, the hash chain protocol offers a limited number of prices from which the bidder can make his bid at. The cost of finding the winning prices depends on the size of the price list. Thus, offering a large list of prices increases the computational cost of the opening phase; meanwhile, offering a small price list reduces the number of price choices for the bidder, and hence reduces the flexibility and the practicality of the protocol.

This paper proposes some modifications to the original hash chain sealed bid auction protocol. These modifications keep all the prominent features that distinguish the original protocol. However, the modified protocol solves most of the problems of the original hash chain protocol. The modified protocol uses the idea of price ranges instead of using discrete prices. This increases the number of price choices available for the bidders and reduces the time needed for the opening phase. During the opening phase of the modified protocol, the auctioneer detects the winning range in which the highest bidding prices exist. The auctioneer then interacts only with the bidders who made their bids within the winning range to specify the winning price and the winning bidder(s) instead of interacting with all the bidders 
participating in the auction. This makes the protocol more user-convenient and decreases the network delay experienced during the opening phase since the auctioneer interacts with a smaller number of bidders.

\section{SEALED-BID AUCTIONS}

\subsection{Definition}

The sealed bid auction is a type of auction in which the buyers are required to submit their bids by a specified deadline. The auctioneer keeps the bid information secret until the deadline, at which time the bids are evaluated and the winners are declared according to a rule: the bidder who bids the highest (or the lowest) price of all the bidders is the winning bidder. Since there is no essential difference between the highest price case and the lowest price case, only the former will be discussed in this paper.

Bidding: Let $\mathrm{A}$ be an auctioneer and $\mathrm{B}_{1}, \mathrm{~B}_{2}, \ldots \ldots, \mathrm{B}_{\mathrm{m}}$. The auctioneer offers some goods for auction (e.g. a painting from a famous painter) and calls the bidders to make their bids for this item. Each bidder $B_{i}$ then decides upon his bidding price and seals this price (e.g. by envelop) and puts his sealed price into auctioneer's ballot box.

Opening: After all the bidders have sealed their bids, the auctioneer opens his ballot box. He reveals each sealed price to find the bidder(s) who bid at the highest price. The bidder(s) who bid at the highest price win the auction and buy the item at the highest price.

\subsection{Security Requirements}

To conduct a fair sealed bid auction, the auction protocol should satisfy the following properties [10]:

1. Secrecy of bidding price: All bidding prices except for the winning price must be kept secret even from the auctioneer. If the auctioneer can obtain some bidding prices before the opening phase, he can collude with other bidders in order to make some bidders win the auction.

2. Verifiability: Anyone should be able to verify the correctness of the auction. Verifiability is an essential requirement to convince the losing bidders of the fairness of the auction.

3. Undeniability: No bidder should be able to deny his bidding price. If a bidder can deny his bid, two bidders can collude to cheat at the auction as follows: Two bidders $B_{1}$ and $B_{2}$ collude and make their bids at prices $P_{1}$ and $P_{2}=P_{1}-1$. If no bidder makes his bid at a price higher than or equal to $P_{1}$, bidder $B_{1}$ denies his bid at price $P_{1}$ and bidder $B_{2}$ wins the 
auction at his price $P_{2}$. If some bidders bid at price $P_{1}$, bidder $B_{1}$ does not deny his bid at price $P_{1}$ and wins the auction at his price $P_{1}$. This means that bidders $B_{1}$ and $B_{2}$ can bid at two selectable prices $P_{1}$ and $P_{2}=P_{1}-1$ and cheat the auction.

4. Anonymity: The bidders should be able to bid anonymously. It is of great importance to keep the privacy of the bidders to prevent collusion between bidders and auctioneer.

\subsection{Previous Work}

In this section, the protocols related to the protocol proposed in this paper are discussed to clarify the effect of the modifications suggested on the efficiency and practicality of the protocol. One of the most relevant protocols is the original hash chain sealed bid auction proposed by Suzuki, Kobayashi, and Morita in [10]. Another relevant protocol is the one proposed by Sako in [5].

\subsubsection{Original Hash Chain Sealed Bid Auction Protocol}

This protocol is the base of the protocol proposed in this paper. It is considered the first sealed bid auction method, which uses only hash functions. In the protocol, the length of the hash chain represents the bidding price, and the bidder uses the head of his hash chain to commit his bid. This protocol satisfies all the requirements of sealed bid auctions. Besides, it drastically reduces the time taken for bidding and opening bids compared to the former methods that purely depend on public key cryptography, for more details about the efficiency of the protocol see [10].

The original hash chain sealed bid auction proceeds as follows:

Preparation: let $\mathrm{A}$ be an auctioneer and $\mathrm{B}_{1}, \mathrm{~B}_{2} \ldots \mathrm{B}_{\mathrm{m}}$ be bidders. Auctioneer A publishes

- $\mathrm{P}=\{1,2 \ldots, \mathrm{n}\}$ : a price list of the auction.

- $\mathrm{h}:\{0,1\}^{\mathrm{st} \mathrm{t}} \longrightarrow\{0,1\}^{\mathrm{t}}:$ a collision intractable random hash function.

- $\mathrm{M}_{\mathrm{no}}, \mathrm{M}_{\mathrm{yes}} \in\{0,1\}^{\mathrm{s}}$ : messages for "I do NOT bid" and "I DO bid".

Bidding: in the bidding phase, each bidder $\mathrm{B}_{\mathrm{i}}$ decides his bidding price $\mathrm{P}_{\mathrm{i}} €$ $P$. he chooses his secret seed $S_{i} \in\{0,1\}^{t}$ randomly and computes a hash chain

$$
\begin{aligned}
& L_{P_{i}-1, i}=S_{i}, L_{P_{i, i}}=h\left(M_{\text {yes }} \| L_{P_{i-1, i}}\right) . \\
& L_{j, i}=h\left(M_{n o} \| L_{j-1, i}\right) \text { where }\left(j=P_{i}+1, P_{i}+2, \ldots, n\right) .
\end{aligned}
$$

$\mathrm{He}$ then sends the commitments of his bidding price with his signature 


$$
\left(\mathrm{L}_{\mathrm{n}, \mathrm{i}}, \operatorname{Sig}_{\mathrm{Bi}}\left(\mathrm{L}_{\mathrm{n}, \mathrm{i}}\right)\right) \text {. }
$$

to auctioneer $A$. auctioneer $A$ receives all bids $\left(\mathrm{L}_{\mathrm{n}, \mathrm{i}}, \operatorname{Sig}_{\mathrm{Bi}}\left(\mathrm{L}_{\mathrm{n}, \mathrm{i}}\right)\right)(\mathrm{i}=$ $1,2, \ldots, \mathrm{m})$, verifies the signatures and publishes all bids.

Opening: in the opening phase, auctioneer A iterates following steps for prices $\mathrm{j}=\mathrm{n}$,

n- $1, \ldots \ldots$

Auctioneer A receives messages $L_{j-1, i}(i=1,2, \ldots, m)$ from all bidders $B_{i}$, and checks the following equality for $i=1,2, \ldots, m$

$$
\mathrm{L}_{\mathrm{j}, \mathrm{i}}=\mathrm{h}\left(\mathrm{M}_{\mathrm{no}} \| \mathrm{L}_{\mathrm{j}-1, \mathrm{i}}\right)
$$

If the above equation holds for all i's, auctioneer $A$ concludes that no bidder bids at price $j$. auctioneer $A$ then publishes all messages $L_{j-1, i}(i=1,2, \ldots, m)$, decreases price $\mathrm{j}$ by 1 , and receives messages $\mathrm{L}_{\mathrm{j}-2, \mathrm{i}}(\mathrm{i}=1,2, \ldots, \mathrm{m})$.

If the above equation does not hold for some i's, auctioneer A checks the following equality for these i's

$$
\mathrm{L}_{\mathrm{j}, \mathrm{i}}=\mathrm{h}\left(\mathrm{M}_{\text {yes }} \| \mathrm{L}_{\mathrm{j}-1, \mathrm{i}}\right)
$$

and concludes that the winning bidders are these $\mathrm{B}_{\mathrm{i}}$ 's and their winning price is $\mathrm{j}$. Finally, auctioneer A publishes the winning bidders, the winning price, and all messages $\mathrm{L}_{\mathrm{j}-1, \mathrm{i}}(\mathrm{i}=1,2, \ldots, \mathrm{m})$.

Notice that messages $M_{n o}$ can be omitted, i.e. each bidder bids $L_{n, i}=h^{n-P i}$ ${ }^{+1}\left(\mathrm{M}_{\mathrm{yes}} \| \mathrm{S}_{\mathrm{i}}\right)$ with his signature.

A sealed bid auction can also be realized with quantity as follows. Each bidder $B_{i}$ decides his bidding equality for each price $i$ and sets the messages

$\mathrm{M}_{\mathrm{j}, \mathrm{i}}=$ "bidding quantity of bidder $\mathrm{B}_{\mathrm{i}}$ at price $\mathrm{j}$ ".

He then makes his hash chain $\mathrm{L}_{\mathrm{j}, \mathrm{i}}=\mathrm{h}\left(\mathrm{M}_{\mathrm{j}, \mathrm{i}} \| \mathrm{L}_{\mathrm{j}-1, \mathrm{i}}\right)$, and bids his commitment with his signature $\left(\mathrm{L}_{\mathrm{n}, \mathrm{i}}, \operatorname{Sig}_{\mathrm{Bi}}\left(\mathrm{L}_{\mathrm{n}, \mathrm{i}}\right)\right)$.

\subsubsection{Universally Verifiable Auction Protocol Which Hides Losing Bids}

Sako proposed a sealed bid auction in which a bid is represented by the encrypted message with the key corresponding to the bidding price. The modified hash chain protocol proposed in this paper uses the ideas suggest in Sako's protocol to solve some of the problems the original hash chain sealed bid auction protocol suffers from. The protocol is briefly described in this section (for more details about the protocol, refer to [5]).

Let $E$ and $D$ be probabilistic encryption and decryption functions. Auctioneer A publishes keys $K_{j}$ and messages $M_{j}$ corresponding to each prices $\mathrm{j} \in\{1,2, \ldots ., \mathrm{n}\}$.

Bidding: Each bidder $\mathrm{B}$ chooses his bidding price $\mathrm{p}$ from the price list $\mathrm{P}$ $=\{1,2, \ldots ., \mathrm{n}\}$. He creates his encrypted message $C_{B i}=E_{K p}\left(M_{p}\right)$ with the key $\mathrm{K}_{\mathrm{p}}$ corresponding to his bidding price $\mathrm{p}$ and publishes it as his bid.

Opening: After all bidders send their bids, the auctioneer iterates following steps for $j=n, n-1, \ldots \ldots$ to open bids.

If relation $D_{\mathrm{Kj}}\left(C_{\mathrm{Bi}}\right)=\mathrm{M}_{\mathrm{j}}$ holds, the auctioneer is convinced that the winning bidder is bidder $B_{i}$ and the winning price is $j$. 
If relation $\mathrm{D}_{\mathrm{Kj}}\left(\mathrm{C}_{\mathrm{Bi}}\right)=\mathrm{M}_{\mathrm{j}}$ does not hold for all bidders, the auctioneer is convinced that no bidder bids at price $\mathrm{j}$. Then, auctioneer $\mathrm{A}$ decreases $\mathrm{j}$ by 1 and repeats the above steps.

This protocol satisfies all the security requirements of sealed bid auctions. Moreover, bidders need not communicate with the auctioneer in the opening phase. However, a malicious auctioneer can reveal all the bidding prices.

\section{THE MODIFIED HASH CHAIN SEALED BID AUCTION}

This protocol is the first to secure the bidding information using a hybrid approach of public key cryptography and one-way hash functions. A hash chain is used to commit the exact bidding price while the range in which this price exists is secured through public key cryptography. This hybrid approach makes the modified hash chain protocol more efficient and practical than the original hash chain protocol that is purely based on oneway hash functions. Furthermore, our approach does not totally depend on public key cryptography, which is very costly. Our approach experiences less network delay at bid opening time and does not require user interaction during bid opening, as in the original hash chain protocol, which increases the practicality and the applicability of the protocol. With practical evidence our protocol has less time complexity and more CPU utilization during the opening phase. These results are shown after describing the protocol.

\subsection{Required Services}

The following services are required by the modified hash chain protocol in order for it to function efficiently:

1. Registration center $(\boldsymbol{R C})$ : this service is required to register bidders before they participate in the auction to assure anonymity. The bidder sends his identity $\mathrm{ID}_{\mathrm{i}}$ (this identity may be his name or any other type of identifiers) along with his public key $\mathrm{Pk}_{\mathrm{Bi}}$ to the registration service. The bidder obtains his public key by generating a pair of public/private keys. The bidder keeps his private key to use it for signing his bidding information while his public key is used by any party who wants to verify the bidder's signature. The registration center then records the pair $\mathrm{ID}_{\mathrm{i}}$ and $\mathrm{Pk}_{\mathrm{Bi}}$ in its database and sends a public key certificate Cert $_{\mathrm{Bi}}$ to the bidder. The public key certificate is used to prove the identity of the bidder without revealing it. This assures the anonymity requirement. 
2. The time service provider: the purpose of the time service provider here is to timestamp each bidder's bid after submitting the bid so that the market can choose the winner in an auction when more than one bidder bids at the same price.

3. The Trusted Third Party (TTP): the role of the TTP is to interact with the auctioneer on behalf of the bidders at bid opening time. The bidder sends the hash chains at each price to the TTP at bidding time. The TTP can then interact with the auctioneer at bid opening time on behalf of the bidders. A discussion about the effect of employing the TTP idea on the practicality of the modified hash chain protocol is provided in section 3.4.

\subsection{Protocol Description}

Registration: In the registration phase, registration center (RC) registers bidder's identifier, public key, and public key certificate to assure anonymity. The time service provider, the Trusted Third Party (TTP) as well as the auctioneer will each register its identity, public key, and public key certificate. The purpose of the time service provider here is to timestamp each bidder's bid so that the market can choose the winner in an auction when more than one bidder bids at the same price.

Step 1: RC confirms the bidder $B_{i}$ who has identifier $\mathrm{ID}_{\mathrm{i}}$ in a safe way. Step 2: $\mathrm{B}_{\mathrm{i}}$ sends public key $\mathrm{Pk}_{\mathrm{Bi}}$ to $\mathrm{RC}$.

Step 3: RC sends public key certificate Cert $_{\mathrm{Bi}}$ to the bidder $\mathrm{B}_{\mathrm{i}}$ and records the pair $\mathrm{ID}_{\mathrm{i}}$ and $\mathrm{Pk}_{\mathrm{Bi}}$ in its database at the same time.

Preparation: let $\mathrm{A}$ be an auctioneer and $\mathrm{B}_{1}, \mathrm{~B}_{2}, \ldots \ldots, \mathrm{B}_{\mathrm{m}}$ be bidders. Let $\mathrm{E}$ and $\mathrm{D}$ be probabilistic encryption and decryption functions. Auctioneer $\mathrm{A}$ publishes:

1. Keys $K_{r}$ and messages $M_{r}$ corresponding to each price range $r €$ $\{1,2, \ldots, z\}$.

2. $P=\{a, a+1, \ldots \ldots \ldots, a+n\}: a$ list of prices within each range; the range starts at price $a$ and ends at price $a+n$ for all ranges 1 ..z. The auctioneer publishes the prices at all ranges.

3. h, h: $\{0,1\}^{\mathrm{st}} \longrightarrow\{0,1\}^{\mathrm{t}}$ : collision intractable random hash functions.

4. $\mathbf{M}_{\mathrm{no}}, \mathrm{M}_{\mathrm{yes}} \in\{0,1\}^{\mathrm{s}}$ : messages for "I do NOT bid" and "I do bid".

\section{Bidding:}

1. In the bidding phase, each bidder $\mathrm{B}_{\mathrm{i}}$ decides upon his price $\mathrm{p}$ and finds the appropriate range $r$ for that price. He signs the range message $M_{r}$ with his private key obtained at reiteration time and produces $\operatorname{Sig}_{\mathrm{Bi}}\left(\mathrm{M}_{\mathrm{r}}\right)$. The bidder then encrypts the signed range 
message with the range public key $\mathrm{K}_{\mathrm{r}}$ that corresponds to his bidding price range $r$ and produces the message $C_{B i}=E_{K r}\left(\operatorname{Sig}_{B i}\left(M_{r}\right)\right)$.

2. The bidder $B_{i}$ chooses his secret seed $S_{i} €\{0,1\}^{t}$ randomly and computes a hash chain

$$
\begin{aligned}
& L_{P i-1}=S_{i} \\
& L_{P i, i}=h\left(M_{y e s} \| L p_{i-1,}, i\right) \\
& L_{j, i}=h\left(M_{n o} \| L_{j-1, i}\right) \quad \text { for }\left(j=P_{i}+1, P_{i}+2, \ldots \ldots, a+n\right) .
\end{aligned}
$$

3. He then sends the $\mathrm{C}_{\mathrm{Bi}}$ along with the commitment of his order price and his signature:

$$
\left(\mathrm{L}_{\mathrm{a}+\mathrm{n}, \mathrm{i}}, \operatorname{Sig}_{\mathrm{Bi}}\left(\mathrm{L}_{\mathrm{a}+\mathrm{n}, \mathrm{i}}\right), \operatorname{Cert}_{\mathrm{Bi}}\right)
$$

to auctioneer $\mathrm{A}$, which then verifies the signature.

4. Auctioneer $\mathrm{A}$ would then send the bidding information to the time service provider to time stamp it.

5. Bidder $B_{i}$ also encrypts $p$ and $r$ using the Trusted Third Party (TTP) public key. $B_{i}$ sends them to the Trusted Third Party (TTP) along with his signature:

$$
\left(E_{T T P}(p), E_{T T P}(r), \operatorname{Cert}_{B i}\right)
$$

6. The TTP would first verify the signature. Then it would decrypt the encrypted price and range and save them along with the bidder ID.

7. Auctioneer A would publish all the bids after receiving all of them.

\section{Opening:}

Step 1: Auctioneer A iterates following steps for ranges $\mathrm{r}=\mathrm{z}, \mathrm{z}-1, \ldots \ldots$ and does the following:

1. Decrypts the range piece of information to obtain the signed range message $\mathrm{D}_{\mathrm{K}}\left(\mathrm{C}_{\mathrm{Bi}}\right)=\operatorname{Sig}_{\mathrm{Bi}}\left(\mathrm{M}_{\mathrm{r}}\right)$

2. Auctioneer $A$ then verifies the signature of bidder $B_{i}$ on the range message $M_{r}$ by doing the following check:

$\operatorname{Ver}_{B i}\left(D_{K}\left(C_{B i}\right)\right)=M_{r}($ for $i=1,2, \ldots \ldots, m)$

If the above equation does not hold for all bidders, auctioneer $\mathrm{A}$ concludes that no bidder bids at range $r$. Thus, $A$ decreases $r$ by 1 and repeat the comparison.

If the above equation holds for some i's, A concludes that those i's bid at prices in that range $r$ and one of them will be the winner of the auction since those bidders are the ones who bid at the highest range. 
Step 2: Auctioneer A iterates following steps for the prices in the range obtained in step $1, j=a_{r}+n, a_{r}+n-1, \ldots \ldots, a_{r}$.

A receives message $L_{j-1, i}$ from the TTP for all i's who bid at that range $r$ (those bidders have been obtained in step 1). A would then check the following equality:

$$
\mathrm{L}_{\mathrm{j}, \mathrm{i}}=\mathrm{h}\left(\mathrm{M}_{\mathrm{no}} \| \mathrm{L}_{\mathrm{j}-1, \mathrm{i}}\right)
$$

If the above equation holds for all bidders who bid at range $\mathrm{r}, \mathrm{A}$ concludes that no bidder bids at price $j$. Auctioneer $A$ then publishes all messages $L_{j-1, i}$ (for all i's obtained in step 1). A would then decrease $\mathrm{j}$ by 1 and repeats the above comparison after receiving $L_{j-2, i}$

If the above equation does not hold for some bidder(s) at some price $\mathrm{j}, \mathrm{A}$ checks the following equality for those bidder(s):

$$
\mathrm{L}_{\mathrm{j}, \mathrm{i}}=\mathrm{h}\left(\mathrm{M}_{\mathrm{yes}} \| \mathrm{L}_{\mathrm{j}-1, \mathrm{i}}\right)
$$

And concludes that the winning bidder(s) are these $\mathrm{B}_{\mathrm{i}}$ 's and their winning price is $\mathrm{j}$. Auctioneer A checks the time stamp for each of those winning bidders so that the bidder who came first wins the auction. Finally auctioneer A publishes the winning bidder(s), the winning price and all messages $L_{j-1, i}$ (for all i's participating in the auction).

\subsection{Protocol Security}

\section{Secrecy of bidding price:}

The auctioneer can easily specify the range at which the winning bidders bid. Since bids are opened from the highest price at that range to the price at which the winning bidders bid, no bids under the winning price are opened. The only piece of information that the auctioneer can know about the losing bidders is the range at which each of them bids but the auctioneer can never know the exact price of any of the losing bidders. Due to the onewayness of the hash function, no one can compute the rest of the hash chain that has not yet been revealed. Due to the randomness of the hash function, no information about the rest of the hash chain that has not yet been revealed can be obtained. Thus, all the bidding prices except the winning price are kept secret even from the auctioneer.

\section{Verifiability:}

Since values of hash chain that are opened are published, anyone can verify the correctness of the hash chain. Due to the collision intractability of the hash function, no one can change the hash chain without changing the committed head of the hash chain. Thus, anyone can verify the correctness of the auction.

\section{Undeniability:}

Since each bid has the bidder's signature, no bidder can deny his bid. Due to the collision intractability of the hash function, no one can change the hash chain without changing the committed head of the hash chain. So no bidder can deny his bidding price. 


\section{Anonymity:}

Because bidding information is constructed using digital signature and public key certificate, and contains no information about bidder's real name, the bidder's real name is not disclosed and each bidder's anonymity to other bidders is preserved.

\subsection{Performance Analysis}

To show the efficiency of our protocol, we compare it to the original hash chain protocol. The comparison includes the cost during the opening phase only since the major differences between the original and the modified hash chain protocol are in the opening phase. A theoretical cost analysis for the opening phase in both the original and the modified hash chain protocol has been performed. This cost is expressed in terms of the time complexity of the opening phase and the network delay experienced during the opening phase. Both cost factors are expressed in big $\mathrm{O}$ notation.

\subsubsection{Time Complexity Comparison}

The time complexity of the opening phase in the original hash chain protocol is $\mathbf{O}($ HashCost $* \mathbf{n m})$, where HashCost is the cost of applying the hash function, $\mathrm{n}$ is the number of prices in the price list, and $\mathrm{m}$ is the entire number of bidders participating in the auction.

The time complexity of the opening phase in the modified hash chain protocol is $\mathbf{O}\left(\right.$ DecCost $\left.^{*} \mathbf{z m}\right)+\mathbf{O}\left(\right.$ HashCost $\left.^{*} \mathbf{n b r}_{\mathrm{w}}\right)$, where the cost parameters are:

1. DecCost: the cost of decrypting the range message at the beginning of the opening phase to detect the winning range.

2. $\mathbf{z}:$ the number of price ranges offered at auction preparation time.

3. m: the total number of bidders participating in the auction.

4. HashCost: the cost of applying the hash function.

5. $\mathbf{n}$ : the number of prices in the winning range.

6. $\mathbf{b r}_{\mathrm{w}}$ : the number of bidders who bid at the winning range.

If those two costs are compared, we will find that the cost of the modified hash chain is less than the cost of the original hash chain. This difference in cost comes from the following:

1. The portion in the cost function of the modified hash chain protocol $\mathbf{O}($ DecCost $* \mathbf{z m})$ does not involve any network delay since the auctioneer goes through all the price ranges sequentially and use their decryption keys till it reaches the highest range at which the bidders has made his bid. Therefore, this factor is not costly and it costs DecCost * $\mathbf{z m}$ in the worst case. However, on average, the auctioneer 
does not go through all the ranges since he stops iterating once he reaches the bidders who bid at the highest range. The network delay needed to exchange the hash chain messages between the auctioneer and the bidders during the opening phase is the most dominant cost factor. Though it is known that the cost of decryption is more than the cost of applying a hash function, the fact that the detection of the winning range in the modified hash chain protocol does not involve any network delay makes the actual time complexity of the modified hash chain protocol less than that of the original protocol especially that in the original protocol, the auctioneer has to remain idle waiting for the bidders to send their hash chains at each price in the price list.

2. The portion of the cost function of the modified hash chain protocol $\mathbf{O}\left(\right.$ HashCost $\left.* \mathbf{n b r}_{\mathbf{w}}\right)$ has a much lower cost than the cost of the original hash chain protocol, which is $\mathbf{O}(\mathbf{n m})$. This cost difference comes from two facts.

i. $\mathbf{n}$ in the cost function of the original hash chain protocol is the number of prices in the price range, which is much larger than the $\mathbf{n}$ in the cost function of the modified hash chain protocol, which is the number of prices in the winning price range only.

ii. $\mathbf{m}$ is the total number of bidders who participate in the auction while $\mathbf{b r}_{\mathbf{w}}$ is the number of bidders who bid in the winning range.

The above point ii makes a difference in the cost of the opening phase of the hash chain protocol and the modified one only if we assume that the distribution of the bidders on the price values is uniform. This assumption means that the probability that a bidder make his bid at a certain price is equal to the probability that the bidder makes his bid at any other price in the price list. This also means that the probability that a bidder makes his bid at a certain price range is equal to the probability that the bidder makes his bid at any other price range, which means that the distribution of the bidders on the price ranges is uniform (this is only valid for the modified hash chain protocol). With this assumption, we can say that in the original hash chain protocol, the auctioneer has to go sequentially through the price list while asking all bidders participating in the auction about their hash chains for each price till it reaches to the winning range. However, for the modified hash chain protocol, we can deduce that the number of bidders who bid at the winning range are much smaller than the total number of participants in the auction since the distribution of the bidders on the bidding prices; and hence on the bidding ranges, is uniform. 


\subsubsection{Network Delay Comparison}

The network delay cost of the opening phase in the original hash chain protocol is $\mathbf{O}(\mathbf{n m x})$, The network delay cost of the opening phase in the modified hash chain protocol is $\mathbf{O}\left(\mathbf{n} \times \mathbf{b}_{\mathrm{rw}}\right)$, where $\mathbf{x}$ (in the cost of both protocols) is the average network delay needed to exchange one message between the bidder and the auctioneer.

As can be noted from the above cost calculation, it can be deduced that the communication cost of the modified hash chain protocol is less than the one of the original hash chain protocol.

If we compare the three parameters in both costs, we will find the following:

- The first parameter in the original hash chain protocol, which is the number of prices in the price list, is larger than the first parameter in the modified hash chain protocol, which is the number of prices in the winning range.

- The second parameter in both the original and the modified hash chain protocols are the same.

- The third parameter in the original hash chain protocol, which is the number of bidders who participate in the auction, is larger than the third parameter in the modified hash chain protocol, which is the number of bidders who bid in the winning range.

- Thus we can conclude that the communication cost of the original hash chain protocol is larger than the communication cost of the modified hash chain protocol.

\subsubsection{Practical Validation}

We did some practical experiments to validate the theoretical cost analysis of the two protocols. Both the original and the modified hash chain protocol have been implemented on the IBM WebSphere Commerce Suite 5.1. This package is an electronic commerce package that includes an auction module. The practical results conform to the theoretical analysis and emphasize it. A summary of the practical results is as follows:

- The original hash chain protocol was slow in evaluating the bidding prices and determining the winners of the auction. The average execution time for the 10 sealed bid auctions for products of medium size is $474.5 \mathrm{sec}$. The opening phase of the original hash chain protocol is slow because the system has to collect the hash chain information from each bidder ( 50 bidders) during each price iteration till it reaches the winning price. The problem with collecting the hash chain is that the system has to broadcast the price at which it wants to collect the hash chain information and waits till the 50 bidders submit their hash chains. 
- The average execution time of the opening phase in the modified hash chain protocol for the same 10 sealed bid auctions is $85.7 \mathrm{sec}$. The average execution time of the opening phase in the original hash chain protocol is $\mathbf{5 . 5 5}$ times the execution time of the opening phase in the modified protocol. This difference in the execution time is due to the fact that during the opening phase of the modified protocol, the system detects the winning range in which the highest bidding price exists easily without any interaction with the bidders. The system then interacts only with the bidders who made their bids in the winning range to detect the winning price and determine the winners of the auction. Since a uniform distribution is employed, the number of bidders in the winning range should not exceed the result of dividing the number of bidders by the number of price ranges. Thus, the number of hash chain messages that should be exchanged between the bidders and the system is reduced radically from the original hash chain protocol.

- The original hash chain sealed bid auction protocol has a low CPU utilization during the opening phase. The average CPU utilization is $43.1 \%$, which is less than half the CPU utilization of the IBM WebSphere Commerce Suite protocol. This low CPU utilization is due to the fact that at each price iteration in the price list, the system has to wait for all the bidders to send their hash chains at that price. Thus, the CPU remains idle waiting for the missing hash chain information, which reduces the efficiency of the protocol.

- The modified hash chain protocol showed a moderate CPU utilization during the opening phase. The average CPU utilization is $71 \%$, which is 1.65 times the CPU utilization of the opening phase in the original hash chain protocol. The improvement in the efficiency of the opening phase of the modified protocol is due to the fact that the system detects the winning range without any interaction with the bidders and then it interacts with only the bidders who made their bids at the winning range. With uniform distribution of bidders on the price ranges, the number of bidders with whom the system interacts in the modified protocol equals the total number of bidders divided by the number of price ranges. Reducing the number of bidders with whom the system interacts increases the CPU utilization, and hence increases the efficiency of the protocol. 
The above practical results show that though the modified hash chain protocol uses public key cryptography along with one-way hash functions, it is 5.55 times faster than the original hash chain protocol that purely depends on one-way hash functions. However, the network delay and the interaction between the bidders and the auctioneer during the opening phase in the original protocol increases the overall time complexity of the opening phase and reduces the efficiency and the practicality of the protocol.

The following 2 graphs show the difference in execution time and in the CPU utilization between the two protocols:

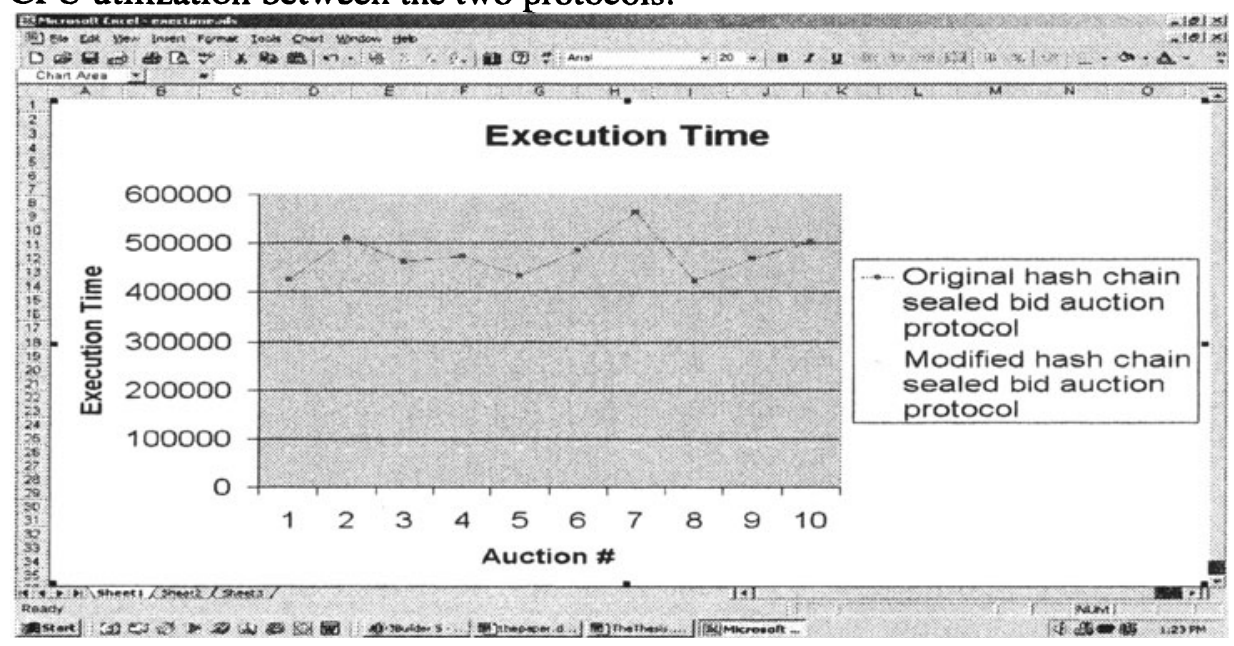

Figure 1: The Relationship between each auction and its execution time for the three protocols



Figure 2: The CPU Utilization of each auction in the three protocols 


\section{CONCLUSION}

We have proposed a sealed bid auction protocol that uses a hybrid approach of the public key cryptography and the one-way hash functions. This protocol is based on another protocol called the original hash chain sealed bid auction protocol, which purely depends on one-way hash functions. Our protocol solves most of the efficiency problems associated with the original hash chain protocol while satisfying all the security requirements of sealed bid auctions. Our protocol reduces the cost of the opening phase due to the fact that it minimizes the network delay experienced during the opening phase.

\section{REFERENCES}

1. Anderson, Ross, "Why Cryptosystems Fail”, University of Cambridge Computer Laboratory, 1999.

2. Cachin, Christian, "Efficient Private Bidding and Auctions with an Oblivious Third Party", IBM research division, Zurich research laboratory, CH 8803 Ruschlikon, Switzerland, 1999.

3. Garfinkel, Simson, and Gene Spefford, "Cryptography and the Web", http://www.cryptography.com 2000.

4. Gorda, Brent, and George V. Wilson, "Building and Running Online Auctions", Dr. Dobb's Journal, Oct. 1997.

5. K. Sako. “Universally verifiable auction protocol which hides losing bids", Proc. of Public Key Cryptography 2000. pp. 35-39, 2000

6. KIKUCHI, Hiroaki, Michael HAKAVY, and Doug TYGAR, "Multi-Round Anonymous Auction Protocols", IEICE Transactions Information \& Systems, Vol. E82-D, NO. 4, April 1999.

7. Kobayashi, Kunio, and H. Morita, "Efficient Sealed Bid Auction Protocol with Quantitative Competition Using One-way Hash Functions", IEICE Technical Report, ISEC99-67, Nov. 1999.

8. Kobayashi, Kunio, et. al., "Efficient Sealed-Bid Auction by Using One-Way Functions", IEICE Transactions Fundamentals, Vol. E84-A, NO. 1, Jan. 2001.

9. Kudo, Michiharu, "Secure Electronic Sealed Bid Auction Protocol with Public Key Cryptography", IEICE Transactions Fundamentals, Vol. E81-A, NO. 1, Jan. 1998.

10. Suzuki, Koutarou, Kunio Kobayashi, and Hikaru Morita, "Efficient Sealed-Bid Auction using hash chain”, NTT Laboratories, Japan, Jan. 2001.

11. Vakrat, Yaniv, and Abraham Seidmann, "Can Online Auctions Beat Online Catalogs", Graduate School of Business Adminstration, Univeristy of Rochester, New York, 2000. 